\title{
The Zeldovich approximation and wide-angle redshift-space distortions
}

\author{
Emanuele Castorina ${ }^{1,2 \star}$, Martin White ${ }^{1,2} \dagger$ \\ ${ }^{1}$ Berkeley Center for Cosmological Physics, University of California, Berkeley, CA 94720, USA \\ ${ }^{2}$ Lawrence Berkeley National Laboratory, 1 Cyclotron Road, Berkeley, CA 93720, USA
}

11 September 2019

\begin{abstract}
The contribution of line-of-sight peculiar velocities to the observed redshift of objects breaks the translational symmetry of the underlying theory, modifying the predicted 2-point functions. These 'wide angle effects' have mostly been studied using linear perturbation theory in the context of the multipoles of the correlation function and power spectrum. In this work we present the first calculation of wide angle terms in the Zeldovich approximation, which is known to be more accurate than linear theory on scales probed by the next generation of galaxy surveys. We present the exact result for dark matter and perturbatively biased tracers as well as the small angle expansion of the configuration- and Fourier-space two-point functions and the connection to the multi-frequency angular power spectrum. We compare different definitions of the lineof-sight direction and discuss how to translate between them. We show that wide angle terms can reach tens of percent of the total signal in a measurement at low redshift in some approximations, and that a generic feature of wide angle effects is to slightly shift the Baryon Acoustic Oscillation scale.
\end{abstract}

\section{INTRODUCTION}

The clustering of galaxies observed in redshift surveys exhibits anisotropies due to the contribution of line-of-sight velocities to the measured redshift Kaiser 1987, Hamilton 1992, 1998, Peacock 1999, Dodelson 2003). This effect, known as redshift space distortions (RSD), enables us to constrain the rate of growth of large-scale structure (Guzzo et al. 2008 Percival \& White 2009), test theories of modified gravity (Joyce et al. 2015) and probe the constituents of the Universe such as massive neutrinos (Lesgourgues \& Pastor 2006, Weinberg et al. 2013).

When modeling the 2-point statistics of redshift-space clustering, most analyses make the "plane parallel approximation", where the direction of the line-of-sight of each object in a pair is assumed to be the same. This means the redshifts of the two objects receive contributions from the same component of the velocity, which increases the symmetry of the system and allows simple Fourier and configuration space analyses. This approximation is usually excellent on small scales and for deep surveys. However for surveys which are relatively shallow and probe large scales - or interferometers whose primary beams cover large sky areas - there are "wide angle" effects which need to be considered. There is a large literature examining these effects within linear perturbation theory (Hamilton 1992; Hamilton \& Culhane 1996a; Hamilton 1998; Zaroubi \& Hoffman 1996; Szalay et al.||1998 | Szapudi||2004, Datta et al.|2007. Pápai \& Szapudi 2008 Shaw \& Lewis 2008 Bonvin \& Dur- rer 2011; Raccanelli et al. 2014 Yoo \& Seljak 2015, Slepian \& Eisenstein 2015, Reimberg et al. 2016 Castorina \& White 2018) but relatively little analytic work beyond linear theory. In this paper we present an analysis of wide angle effects to lowest order in Lagrangian perturbation theory, i.e. the Zeldovich approximation (ZA; Zel'dovich 1970). The ZA is quite accurate on large scales, and provides a better modeling of features in the spectrum such as baryon acoustic oscillations than does linear theory (Tassev 2014a). It also contains more angular structure than is present in linear theory, allowing us to examine in more detail the impact of relaxing the plane parallel approximation. A calculation of non-linear wide angle terms in standard (Eulerian) perturtabion theory beyond linear theory was presented in Shaw \& Lewis (2008). Our analysis differs from theirs in that we resum the linear displacement fields and include the effect of biasing of halos and galaxies with respect to the dark matter field.

In this work we shall focus on the 'physical' wide angle terms, arising from the projection of peculiar velocities onto a line of sight that varies across the sky, and their effect on the non linear dynamics of the matter and galaxy density fields. It is well known that a survey's selection function will introduce extra wide angle terms (Kaiser 1987, Hamilton \& Culhane 1996b, Szalay et al. 1998, Castorina \& White 2018). The latter have been studied in linear perturbation theory, but we are not aware of any formalism to include them beyond this regime. However if the selection function is slowly varying with redshift we expect higher order terms to be small. 


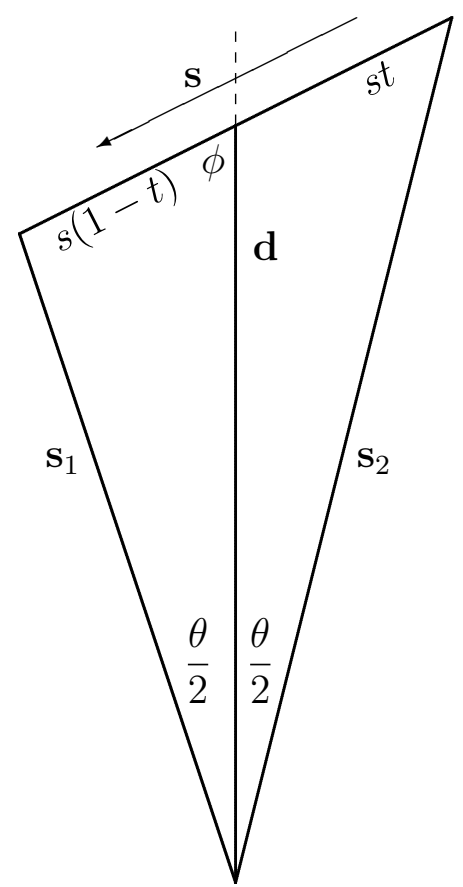

Figure 1. The assumed geometry and angles. The two galaxies lie at $\mathbf{s}_{1}$ and $\mathbf{s}_{2}$, with separation vector $\mathbf{s}=\mathbf{s}_{1}-\mathbf{s}_{2}$ and enclosed angle $\theta$. We take the line of sight to be parallel to the angle bisector, d, which divides $\mathbf{s}$ into parts of lengths $s t$ and $s(1-t)$. The separation vector, $\mathbf{s}$, makes an angle $\phi$ with the line of sight direction, $\hat{d}$.

The outline of the paper is as follows. In \$2 we review the formalism of redshift-space distortions and the wide angle effects and outline the geometry of the problem, including the bisector and end-point conventions for defining the line of sight. In this section we also provide an explicit connection between the 3D correlation function and power spectrum multipoles and the multi-frequency angular power spectrum (MAPS), showing explicitly that the angular structure arises through projection and allowing computation of the MAPS beyond the plane-parallel approximation. In $\$ 3$ we describe how to include wide angle effects into the ZA for both the correlation function (Tassev 2014b) and the power spectrum. The full result is evaluated numerically in 3.1 , whereas in $\$ 3.2$ we present a small angle expansion and the relation between the $\mathrm{ZA}$, linear theory and the different conventions for the line of sight. We conclude in 4 and discuss some technical details in the appendices.

\section{WIDE ANGLE EFFECTS}

The study of "wide angle" effects in galaxy clustering has a long history. Here we briefly review some important background material and make connections between different conventions for the line of sight and different 2-point clustering statistics. We shall adopt the notation and conventions of Castorina \& White (2018), which builds upon the earlier work of Hamilton \& Culhane (1996a); Zaroubi \& Hoffman (1996); Szalay et al. (1998) and Pápai \& Szapudi (2008).

\subsection{Geometry}

The geometry of a generic redshift space configuration for the 2-point function is shown in Fig. 2 Three numbers are enough to properly describe the system, and several possible choice of parametrization are available. The observer $O$ is looking at two objects at $\mathbf{s}_{1}$ and $\mathbf{s}_{2}$, separated by $\mathbf{s}=\mathbf{s}_{1}-\mathbf{s}_{2}$ and the line of sight (LOS) $\mathbf{d}$ is defined as the bisector of the angle $\theta$ between $\mathbf{s}_{1}$ and $\mathbf{s}_{2}$. The LOS and the separation vector form an angle $\phi$, with $\cos (\phi) \equiv \mu$. We also define $t \in[0,1]$ through

$$
\mathbf{s}_{1}=\mathbf{d}+(1-t) \mathbf{s} \quad, \quad \mathbf{s}_{2}=\mathbf{d}-t \mathbf{s} .
$$

The clustering is independent of the direction of $\mathbf{d}$ and of rotations of $\mathbf{s}$ about $\mathbf{d}$, thus the correlation function can be written as a function of $s, \mu$ and $d$. We follow Reimberg et al. (2016) and define a small parameter $x \equiv s / d \ll 1$, as this will prove convenient later.

Castorina \& White (2018) show that for the configuration in Fig. 2

$$
\mathbf{d}=\frac{s_{1} s_{2}}{s_{1}+s_{2}}\left(\hat{s}_{1}+\hat{s}_{2}\right) \quad, \quad d^{2}=\frac{4 s_{1}^{2} s_{2}^{2}}{\left(s_{1}+s_{2}\right)^{2}} \cos ^{2} \frac{\theta}{2}
$$

and

$$
1-t=\frac{-1+\mu x+\sqrt{1+\mu^{2} x^{2}}}{2 \mu x} \simeq \frac{1}{2}+\frac{\mu x}{4}-\frac{\mu^{3} x^{3}}{16}+\cdots
$$

Another popular choice of coordinate system, which we call the end-point parametrization, is to use one of the two galaxies, for instance $\mathbf{s}_{1}$, as the line of sight. This is mostly motivated by the fact that in this case estimators for the power spectrum multipoles can be written as simple Fast Fourier Transforms (FFT) (Scoccimarro 2015, Bianchi et al. 2015, Hand et al. 2017). On the other hand one loses the reflection symmetry around the observer, i.e. the redshift space correlation function is not invariant under $\mathbf{s}_{1} \rightarrow-\mathbf{s}_{1}$. For this choice of LOS wide angle effects are much bigger than for the midpoint or the bisector, and odd multipoles are also generated (Reimberg et al. 2016, Castorina \& White 2018). It is relatively straightforward to go from one parametrization to another one using

$$
\begin{aligned}
\hat{s}_{1} \cdot \hat{s}_{2} & \simeq 1-\frac{x_{1}^{2}}{2}\left(1-\mu_{1}^{2}\right)+\cdots \\
\mu & \simeq \mu_{1}-\frac{x_{1}}{2}\left(1-\mu_{1}^{2}\right)+\cdots \\
x & \simeq x_{1}\left(1+\frac{\mu_{1} x_{1}}{2}\right)+\cdots
\end{aligned}
$$

with $\mu_{1}=\hat{s} \cdot \hat{s}_{1}$ and expansion parameter $x_{1}=s / s_{1} \ll 1$. We shall develop the formalism within the bisector convention and discuss how to transform to the end-point convention where appropriate.

\subsection{Correlation function}

It is common to express the correlation function as an expansion in $\mu$ via:

$$
\begin{aligned}
\xi_{s}(s, d, \mu) & =\sum_{\ell} \xi_{\ell}(s, d) \mathcal{L}_{\ell}(\mu) \\
& =\sum_{\ell n} x^{n} \xi_{\ell}^{(n)}(s) \mathcal{L}_{\ell}(\mu)
\end{aligned}
$$


where $\mathcal{L}_{\ell}$ indicates the Legendre polynomial of order $\ell$ and in the second line we have expanded $\xi_{\ell}(s, d)$ in powers of the wide angle parameter, $x=s / d$. The lowest order terms are the familiar "plane parallel" approximation. In linear theory (Hamilton 1992)

$$
\begin{aligned}
& \xi_{0}^{p p}(s)=\xi_{0}^{(0)}(s)\left(1+\frac{2}{3} f+\frac{1}{5} f^{2}\right) \\
& \xi_{2}^{p p}(s)=\xi_{2}^{(0)}(s)\left(-\frac{4}{3} f-\frac{4}{7} f^{2}\right) \\
& \xi_{4}^{p p}(s)=\xi_{4}^{(0)}(s)\left(\frac{8}{35} f^{2}\right)
\end{aligned}
$$

with

$$
\xi_{\ell}^{(0)}(s)=\int \frac{k^{2} d k}{2 \pi^{2}} P(k) j_{\ell}(k s)
$$

and $P(k)$ the linear theory power spectrum.

\subsection{Power spectrum}

The definition of the redshift space power spectrum requires some care if we drop the plane-parallel approximation (Zaroubi \& Hoffman 1996). What is always well-defined is the "local", i.e. LOS-dependent, power spectrum (Scoccimarro 2015, Reimberg et al. 2016),

$$
P(\mathbf{k}, \mathbf{d}) \equiv \int \mathrm{d}^{3} s \xi(\mathbf{s}, \mathbf{d}) e^{-i \mathbf{k} \cdot \mathbf{s}}
$$

which can be expanded in multipoles as

$$
\begin{aligned}
P(\mathbf{k}, \mathbf{d}) & =\sum_{\ell} P_{\ell}(k, d) \mathcal{L}_{\ell}(\hat{k} \cdot \hat{d}) \\
& \equiv \sum_{n}(k d)^{-n} P_{\ell}^{(n)}(k) \mathcal{L}_{\ell}(\hat{k} \cdot \hat{d})
\end{aligned}
$$

In this case multipoles of the correlation function and of the power spectrum are still related by a Hankel-transform,

$$
P_{\ell}(k, d)=4 \pi(-i)^{\ell} \int s^{2} \mathrm{~d} s j_{\ell}(k s) \xi_{\ell}(s, d)
$$

and thus

$$
P_{\ell}^{(n)}(k)=4 \pi(-i)^{\ell} \int s^{2} \mathrm{~d} s(k s)^{n} \xi_{\ell}^{(n)}(s) j_{\ell}(k s)
$$

with inverse

$$
\xi_{\ell}^{(n)}(s)=\int \frac{k^{2} \mathrm{~d} k}{2 \pi^{2}}(k s)^{-n} P_{\ell}^{(n)}(k) j_{\ell}(k s) .
$$

In observations the most commonly used estimator for the power spectrum multipoles has been proposed by Yamamoto et al. (2006):

$$
\begin{aligned}
\hat{P}_{L}^{Y}(k) & \equiv \frac{(2 L+1)}{V} \int \frac{\mathrm{d} \Omega_{\mathbf{k}}}{4 \pi} \mathrm{d}^{3} s_{1} \mathrm{~d}^{3} s_{2} \\
& \times \delta\left(\mathbf{s}_{1}\right) \delta\left(\mathbf{s}_{2}\right) e^{-i \mathbf{k} \cdot \mathbf{s}} \mathcal{L}_{L}(\hat{k} \cdot \hat{d})
\end{aligned}
$$

where $V$ is the survey volume, and the line of sight can be either the bisector or the midpoint. Taking the expectation value of this estimator yields (Castorina \& White 2018)

$$
\begin{aligned}
\left\langle\hat{P}_{L}^{Y}(k)\right\rangle & =(2 L+1) \int \frac{\mathrm{d}^{3} d}{V} \frac{\mathrm{d} \Omega_{\mathbf{k}}}{4 \pi} P(\mathbf{k}, \mathbf{d}) \mathcal{L}_{L}(\hat{k} \cdot \hat{d}) \\
& =\int \frac{\mathrm{d}^{3} d}{V} P_{L}(k, d)
\end{aligned}
$$

At lowest order in $x$ the expectation value of $\hat{P}_{L}^{Y}$ is simply $P_{\ell}^{(0)}(k)$ as in Eq. 15 .

For computational reasons the estimator is more commonly defined using the direction to one of the two galaxies as the line of sight

$$
\begin{aligned}
\hat{P}_{L}^{F F T}(k) & \equiv \frac{(2 L+1)}{V} \int \frac{\mathrm{d} \Omega_{\mathbf{k}}}{4 \pi} \mathrm{d}^{3} s_{1} \mathrm{~d}^{3} s_{2} \\
& \times \delta\left(\mathbf{s}_{1}\right) \delta\left(\mathbf{s}_{2}\right) e^{-i \mathbf{k} \cdot \mathbf{s}} \mathcal{L}_{L}\left(\hat{k} \cdot \hat{s}_{1}\right)
\end{aligned}
$$

which can be evaluated using FFTs (Scoccimarro 2015 Bianchi et al. 2015, Hand et al. 2017), compared to brute force pair sum required in Eq. (17). The ensemble average of the FFT estimator can be related to the multipoles of the theoretical correlation function defined using the bisector or the end-point as the line of sight following Castorina \& White (2018), who have also shown that wide angle corrections to Eq. 20 are in general much bigger than the one of Eq. 17). We shall denote the coefficients of the expansion of the end-point-based $P_{\ell}\left(k, s_{1}\right)$ in powers of $\left(k s_{1}\right)$ as $\tilde{P}_{\ell}^{(n)}(k)$ to distinguish them from the bisector-based $P_{\ell}^{(n)}(k)$ of Eq. 13.

\subsection{Fourier-Bessel expansion and MAPS}

A third representation of the 2-point function is in terms of the Fourier-Bessel (sFB) expansion (Lahav et al. 1994, Fisher et al. 1994, Heavens \& Taylor 1995 Percival et al. 2004 Padmanabhan et al. 2001 Pratten \& Munshi||2013. Castorina \& White 2018) or its configuration space analog, the multi-frequency angular power spectrum (MAPS; Datta et al. 2007. see also Castorina \& White 2018). The latter has most commonly been used to describe fluctuations measured by wide-area, interferometric, $21 \mathrm{~cm}$ instruments (e.g. Shaw et al. 2014).

In this formalism, one describes the triangle of Fig. 2 in terms of the two side lengths $\left(s_{1}\right.$ and $\left.s_{2}\right)$ and the enclosed angle $(\theta)$. Expanding the $\theta$-dependence in Legendre polynomials

$$
\xi\left(\mathbf{s}_{1}, \mathbf{s}_{2}\right)=\sum_{\ell=0}^{\infty} \frac{2 \ell+1}{4 \pi} C_{\ell}\left(s_{1}, s_{2}\right) \mathcal{L}_{\ell}(\cos \theta)
$$

the coefficients, $C_{\ell}$, are the MAPS and their one dimensional Hankel transform along $s_{1}$ and $s_{2}$ the angular power spectra

$$
C_{\ell}\left(k_{1}, k_{2}\right)=\int s_{1}^{2} \mathrm{~d} s_{1} \int s_{2}^{2} \mathrm{~d} s_{2} C_{\ell}\left(s_{1}, s_{2}\right) j_{\ell}\left(k_{1} s_{1}\right) j_{\ell}\left(k_{2} s_{2}\right) \text {. }
$$

So far most treatments of spherical power spectra have been confined to linear theory, limiting their use in real data where one has to deal with e.g. Fingers-of-God (FOG) (Percival et al. 2004) and non-linearities in the galaxy field (Pratten \& Munshi 2013). This was due to the very complicated expression the spherical coefficients of the density fields take beyond linear theory.

However, we know from the 3D Cartesian analysis that at large scales most of the power is confined in a few multipoles, $L$, and thus the complex structure of the MAPS or angular power spectra must predominantly result from projection effects. This is similar to what happens with the CMB where only a few multipoles are relevant at recombination and the rich structure we observe today is due to LOS 
projection (Dodelson 2003). Let us try to see this directly from Eqs. 21 22 . Inverting Eq. 21

$$
C_{\ell}\left(s_{1}, s_{2}\right)=2 \pi \int_{-1}^{1} \mathrm{~d}(\cos \theta) \xi\left(\mathbf{s}_{1}, \mathbf{s}_{2}\right) \mathcal{L}_{\ell}(\cos \theta)
$$

we see that if we approximate $\mathcal{L}_{\ell}(\cos \theta)$ with $J_{0}(\ell \theta)$, valid for $\theta \ll 1$, then the $C_{\ell}\left(s_{1}, s_{2}\right)$ is the LOS Fourier transform of $P(\mathbf{k})$ with $\ell=k_{\perp} d$ (Appendix A).

In general the MAPS can be expressed as an integral over the power spectrum. The full expression 1 using the bisector definition of $\mathbf{d}$ is extremely cumbersome, but it simplifies dramatically if we instead use the end-point definition. Expanding the exponentials using the Rayleigh expansion of the plane wave and combining products of spherical harmonics using the Gaunt integral one can show

$$
\begin{aligned}
& 2 \pi \int \mathrm{d}(\cos \theta) \mathcal{L}_{\ell}(\cos \theta) \int \frac{\mathrm{d} \Omega_{k}}{4 \pi} e^{i \mathbf{k} \cdot\left(\mathbf{s}_{1}-\mathbf{s}_{2}\right)} \mathcal{L}_{L}\left(\hat{k} \cdot \hat{s}_{1}\right) \\
& =4 \pi \sum_{\lambda}(2 \lambda+1) i^{\lambda-\ell}\left(\begin{array}{ccc}
\lambda & \ell & L \\
0 & 0 & 0
\end{array}\right)^{2} j_{\lambda}\left(k s_{1}\right) j_{\ell}\left(k s_{2}\right) .
\end{aligned}
$$

Thus our $C_{\ell}$ become

$$
\begin{aligned}
C_{\ell}\left(s_{1}, s_{2}\right) & =2 \pi \int \mathrm{d}(\cos \theta) \mathcal{L}_{\ell}(\cos \theta) \int \frac{\mathrm{d}^{3} k}{(2 \pi)^{3}} e^{i \mathbf{k} \cdot\left(\mathbf{s}_{1}-\mathbf{s}_{2}\right)} \\
& \times \sum_{L} P_{L}\left(k, s_{1}\right) \mathcal{L}_{L}\left(\hat{k} \cdot \hat{s}_{1}\right) \\
& =\sum_{L \lambda} F_{L \lambda}^{\ell} \int \frac{k^{2} \mathrm{~d} k}{2 \pi^{2}} P_{L}\left(k, s_{1}\right) j_{\lambda}\left(k s_{1}\right) j_{\ell}\left(k s_{2}\right)
\end{aligned}
$$

where

$$
F_{L \lambda}^{\ell}=4 \pi(2 \lambda+1) i^{\lambda-\ell}\left(\begin{array}{ccc}
\ell & L & \lambda \\
0 & 0 & 0
\end{array}\right)^{2}
$$

The triangle condition of the $3 j$-coefficients makes the sum over $\lambda$ finite, as $|\lambda-\ell| \leqslant L$.

Note that we did not have to assume any specific model for the power spectrum: Eq. 25 can be used to describe any model of the angular correlation function. The final expression is remarkably simple and shows how the structure at $\ell>L$ is only due to projection along the direction to the two galaxies. The expression for the angular power spectrum can be written in an even simpler form,

$$
C_{\ell}\left(k_{1}, k_{2}\right)=\sum_{L \lambda} \frac{F_{L \lambda}^{\ell}}{4 \pi} \int s^{2} \mathrm{~d} s P_{L}\left(k_{2}, s\right) j_{\ell}\left(k_{1} s\right) j_{\lambda}\left(k_{2} s\right)
$$

and expanding $P_{L}\left(k_{2}, s\right)$ in powers of $\left(k_{2} s\right)$ Reimberg et al. 2016, Castorina \& White 2018)

$$
\begin{aligned}
C_{\ell}\left(k_{1}, k_{2}\right) & =\sum_{L \lambda n} \frac{F_{L \lambda}^{\ell}}{4 \pi} \tilde{P}_{L}^{(n)}\left(k_{2}\right) \int s^{2} \mathrm{~d} s\left(k_{2} s\right)^{-n} j_{\ell}\left(k_{1} s\right) j_{\lambda}\left(k_{2} s\right) \\
& \equiv \sum_{L \lambda n} \mathcal{M}_{\ell, L, \lambda}^{(n)}\left(k_{1}, k_{2}\right) \tilde{P}_{L}^{(n)}\left(k_{2}\right)
\end{aligned}
$$

Hence the sFB power spectrum is the product of the (3D) multipoles of the power spectrum times a geometric term

\footnotetext{
1 One proceeds by using the addition theorem and Rayleigh expansion of the plane wave, then expands the $Y_{\ell m}$ using solid harmonics as in Appendix E of Castorina \& White (2018), and simplifies the angular integrals using $3 j$-coefficients.
}

that can be expressed ${ }^{2}$ in terms of hypergeometric functions and does not depend on any cosmological parameters. Note for small $L$ and large $\ell$ the $F_{L \lambda}^{\ell}$ are non-zero only for $\lambda \approx \ell$ and the integral is highly peaked around $k_{1}=k_{2}{ }^{3}$ The above expressions provide an exhaustive description of the two point statistics for redshift surveys in spherical coordinates, completing the description of Liu et al. (2016) for Intensity Mapping surveys and of Passaglia et al. (2017) for imaging surveys, neither of which included redshift space distortions. The other main issue in spherical analysis is the estimate of the covariance matrix. Since $\ell$ can easily go up to a few hundreds and the power spectrum is estimated in tens of $k$-bins, the dimensionality of the covariance makes the problem very quickly intractable (Percival et al. 2004). Eqs. 25 29) offer a simple solution to this problem, as the $C_{\ell}$ and the $P_{L}$ are linearly related to each other by a matrix that can be 'inverted' to find an optimal data compression. Given a survey geometry and galaxy selection function one needs to measure only the $\ell, k_{1}, k_{2}$ that maximize the signal and at the same time keep the dimensionality of the problem low enough. We finally point out that Eq. 29 provides an elegant and unbiased way to remove systematics in the plane of the sky, e.g. fiber collision in spectroscopic instruments (Hahn et al. 2017), that by definition affect only the low- $k_{\|}$modes. The wave numbers appearing on the left-hand side are indeed radial Fourier modes, whereas the ones of the right-hand side are 3D Cartesian modes.

\section{ZELDOVICH APPROXIMATION}

Almost all prior work on wide angle effects used an Eulerian, linear theory description of the 2-point function. Instead we shall base our analytic model of wide angle effects on $1^{\text {st }}$ order Lagrangian perturbation theory - the ZA (Zel'dovich 1970). Despite the more than 40 years since it was first introduced, the ZA still provides one of our most accurate models for the distribution of cosmological objects. It has been applied to understanding the impact of non-linearities on BAO (Padmanabhan et al.|2009 |Noh et al.|2009, McCullagh \& Szalay 2012 |Tassev \& Zaldarriaga 2012a), to reconstruction (Tassev \& Zaldarriaga |2012b;|White|2015), as the basis of an effective field theory (Porto et al. 2014 Vlah et al. 2015) and as a rapid means of simulating large-scale structure (Doroshkevich et al. 1980, Coles et al. 1993, Pauls \& Melott 1995 Sahni \& Coles 1995 Hidding et al. 2014. Chuang et al. 2015). The ZA can easily incorporate wide angle effects, and is quite accurate on the large scales where such effects are most important. For a pedagogical introduction to the ZA and the analytic calculation of the correlation function see e.g. Carlson et al. (2013); White (2014); Tassev (2014b).

Following the usual approach we denote the Lagrangian position of a fluid element by $\mathbf{q}$, and its final (Eulerian) position by $\mathbf{x}=\mathbf{q}+\boldsymbol{\Psi}(\mathbf{q}, t)$. This serves to define the displacement, $\boldsymbol{\Psi}$. To lowest order in perturbation theory, i.e. the

2 The relevant expression is on p. 401 (\$13.4) of Watson (1966).

${ }^{3}$ For $\ell \neq \lambda$ and $n=0$ the off-diagonal terms in Eq. 29] decay as $\min \left[\left(k_{1} / k_{2}\right)^{\ell},\left(k_{2} / k_{1}\right)^{\lambda}\right]$ when $\ell$ and $\lambda$ are large. 


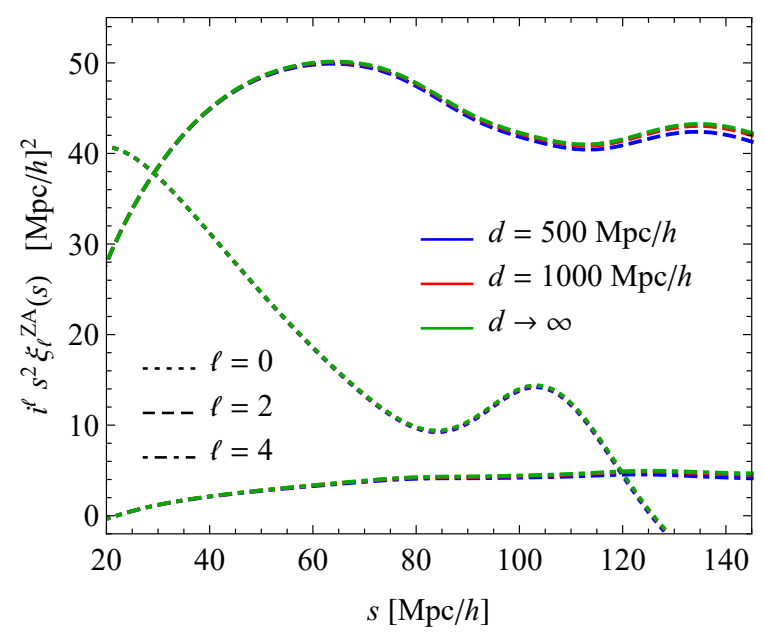

Figure 2. The multipoles of the redshift-space, matter correlation function $(b=1)$, computed from Eq. $(36$, with the bisector definition of the line-of-sight, for $d=500 h^{-1} \mathrm{Mpc}$ (blue), $1 h^{-1} \mathrm{Gpc}$ (red) and $10 h^{-1} \mathrm{Gpc}$ (green). The differences are barely visible on this linear scale and the $10 h^{-1} \mathrm{Gpc}$ lines form an excellent approximation to the plane-parallel or small-angle limit $(d \rightarrow \infty)$.

$\mathrm{ZA}$,

$$
\Psi(\mathbf{q})=\int \frac{d^{3} k}{(2 \pi)^{3}} e^{i \mathbf{k} \cdot \mathbf{q}} \frac{i \mathbf{k}}{k^{2}} \delta_{L}(\mathbf{k})
$$

Within the ZA the transition to redshift space is straightforward, and indeed this is one of the major advantages of Lagrangian perturbation theory in large-scale structure. For an object observed in direction $\hat{s}$ the redshift-space displacement is related to the real-space displacement by

$$
\mathbf{\Psi}(\mathbf{q}) \rightarrow \mathbf{R} \Psi(\mathbf{q})=(1+f \hat{s} \hat{s}) \Psi(\mathbf{q})
$$

For our purposes the important point to note is that $\hat{s}$ is $q$-independent. This means that it is fixed in the integrals over $d^{3} q$ that define the density field and correlation function (see below) which makes the inclusion of wide-angle effects within the ZA very straightforward (Tassev 2014b).

If we make the standard definitions that $\boldsymbol{\Delta}=\boldsymbol{\Psi}\left(\mathbf{q}_{1}\right)-$ $\mathbf{\Psi}\left(\mathbf{q}_{2}\right)$ where $\mathbf{q}=\mathbf{q}_{1}-\mathbf{q}_{2}$ and $A_{i j}=\left\langle\Delta_{i} \Delta_{j}\right\rangle=X(q) \delta_{i j}+$ $Y(q) \hat{q}_{i} \hat{q}_{j}, U_{i}=\left\langle\Delta_{i} \delta_{2}\right\rangle=U(q) \hat{q}_{i}, \xi=\left\langle\delta_{1} \delta_{2}\right\rangle$ then

$$
\begin{aligned}
X(q) & =\int \frac{d k}{2 \pi^{2}} P(k)\left[\frac{2}{3}-2 \frac{j_{1}(k q)}{k q}\right] \\
Y(q) & =\int \frac{d k}{2 \pi^{2}} P(k)\left[-2 j_{0}(k q)+6 \frac{j_{1}(k q)}{k q}\right] \\
U(q) & =\int \frac{d k}{2 \pi^{2}} P(k)\left[-k j_{1}(k q)\right] \\
\xi(q) & =\int \frac{d k}{2 \pi^{2}} P(k)\left[k^{2} j_{0}(k q)\right]
\end{aligned}
$$

where $P(k)$ is the linear theory power spectrum, and the

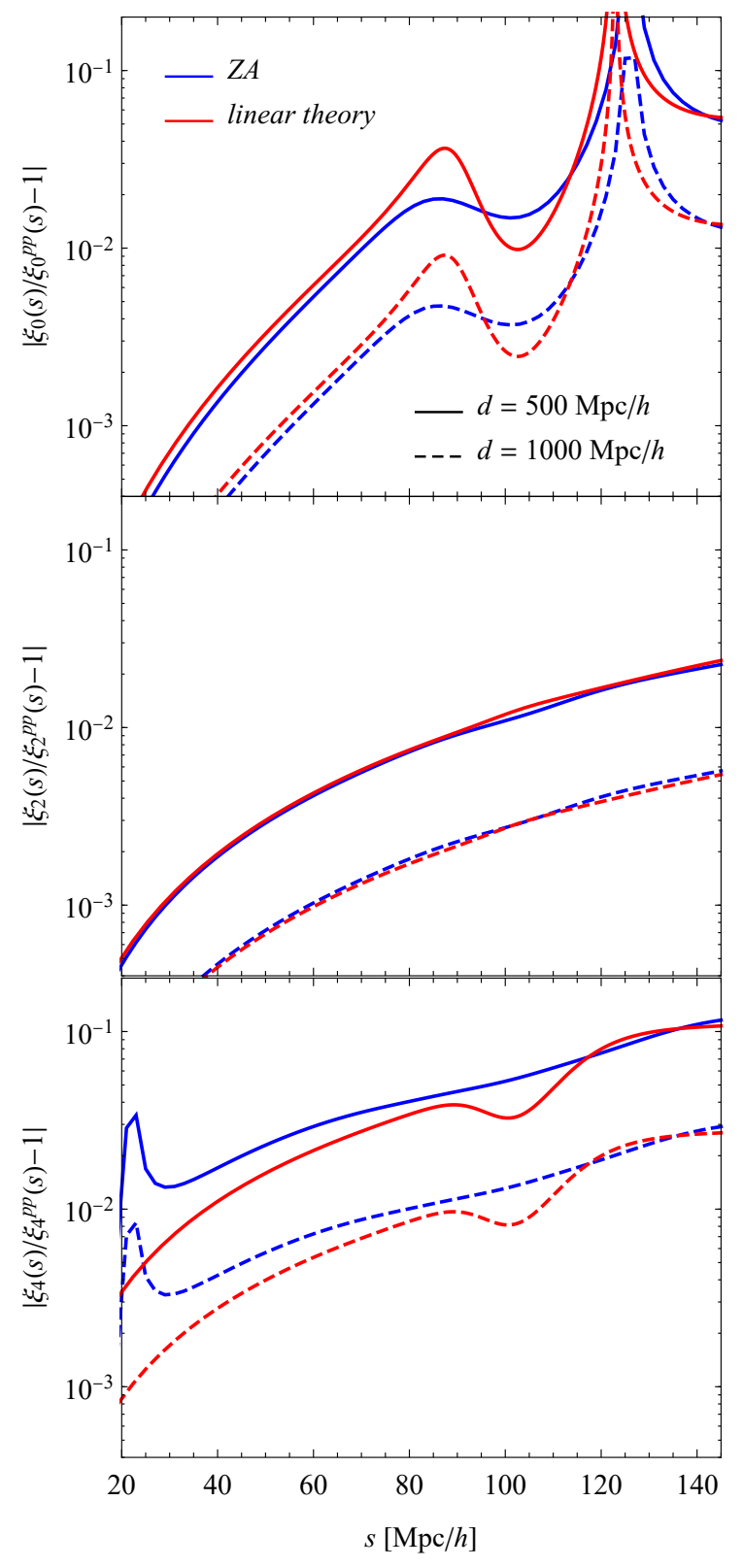

Figure 3. The ratio of the multipoles of the redshift-space, matter correlation function $\left(b_{1}=0\right.$ or $\left.b=1\right)$ for $d=500 h^{-1} \mathrm{Mpc}$ (solid) and $1 h^{-1} \mathrm{Gpc}$ (dashed) to the same multipoles in the plane parallel approximation (Eq. 9p. The blue lines show the ZA (Eq. 36) while the red lines show linear theory (from Castorina \& White 2018).

redshift-space correlation function can be written as

$$
\begin{aligned}
1+\xi^{(s)}(s, d, \mu) & =\int d^{3} q \frac{d^{3} k}{(2 \pi)^{3}} e^{i k_{i}(q-s)_{i}} e^{-(1 / 2) k_{i} A_{s, i j} k_{j}} \\
& \times\left[1+2 i b_{1} k_{i} U_{s, i}+b_{1}^{2} \xi+\mathcal{O}\left(P_{L}^{2}\right)\right] \\
& =\int \frac{d^{3} q}{(2 \pi)^{3 / 2}\left|A_{s}\right|^{1 / 2}} e^{-(1 / 2)(\mathbf{q}-\mathbf{s}) A_{s}^{-1}(\mathbf{q}-\mathbf{s})} \\
& \times\left[1-2 b_{1} g_{i} U_{i}+b_{1}^{2} \xi+\mathcal{O}\left(P_{L}^{2}\right)\right]
\end{aligned}
$$




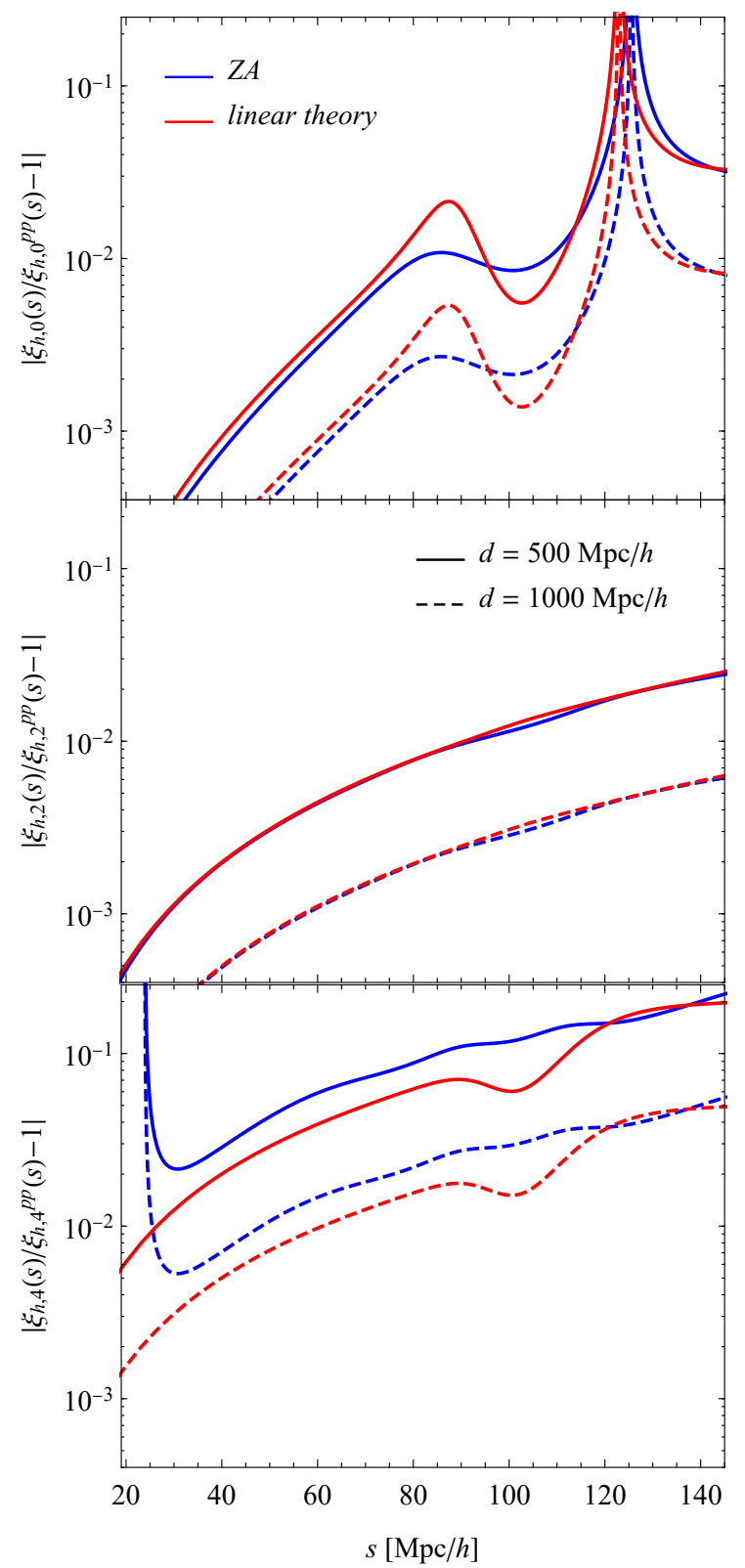

Figure 4. Same as Fig. 3 but now for tracers with linear bias $b=2$ (i.e. $\left.b_{1}=1\right)$.

where

$$
\begin{aligned}
A_{s} & =\left(R_{1}^{2}+R_{2}^{2}\right) \Sigma^{2} \delta_{i j}+\left(R_{1} \xi R_{2}+R_{2} \xi R_{1}\right) \\
A_{s} & =\frac{1}{2}\left(R_{1} A R_{2}+R_{2} A R_{1}\right) \\
g_{i} & =A_{s, i j}^{-1}\left(q_{j}-s_{j}\right) \\
U_{s, i} & =\frac{1}{2}\left(R_{1, i j}+R_{2, i j}\right) U_{j}
\end{aligned}
$$

In the above we have written the real space displacement correlation function as $A_{i j}=2 \Sigma^{2} \delta_{i j}+2 \xi_{i j}$ and included biased tracers to lowest order in the Lagrangian bias, following Matsubara (2008). This will be sufficient for our purposes (see Matsubara 2008, Carlson et al. 2013, White 2014 or Vlah et al. |2016 for the higher-order terms). The large

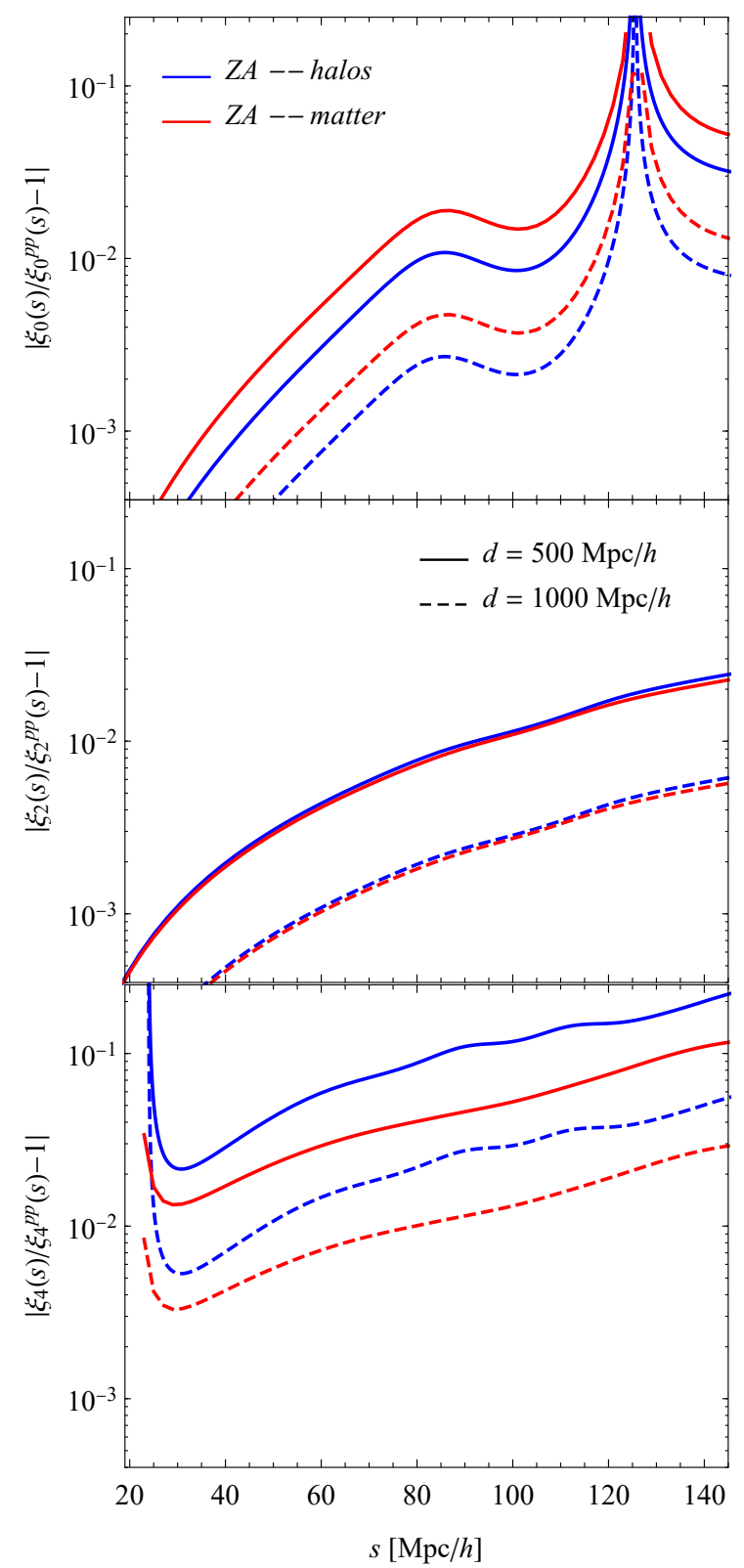

Figure 5. Comparison between the size of the wide angle terms (in the ZA) for dark matter (red) and tracers with $b=2$ (blue).

scale, Eulerian bias is simply related to our Lagrangian bias parameter $\left(b_{1}\right)$ through $b=1+b_{1}$.

At this point we have two routes forward, and we shall discuss them in the next two subsections. Section 3.1 presents a numerical calculation, whereas in Section 3.2 we will expand the Zeldovich calculation to $\mathcal{O}\left(x^{2}\right)$ to make contact with the linear theory solution.

\subsection{Numerical evaluation}

The first approach is to simply evaluate the integral in Eq. 36 numerically. We start by considering the bisector definition of $\hat{d}$. Placing $\hat{d}$ along the $\hat{z}$-axis and orienting $\hat{s}_{1}$ and $\hat{s}_{2}$ in the $x-z$ plane the $3 \mathrm{D}$ integral is well behaved and converges rapidly for any triangle configuration. A further 
numerical integral over $\mu$ at fixed $s$ and $d$ then gives $\xi_{\ell}(s, d)$. We show this in Fig. 2 for $\ell=0,2$ and 4 for several values of $d$. In order to isolate the wide-angle behavior from the evolving dynamics we have assumed (unphysically) a fixed $\Lambda$ CDM model at $z=0.25$ for each of these situations and set $b_{1}=0$. In reality increasing depth $(d)$ would also change the mean redshift, the growth and the degree of non-linearity. These effects can all be accounted for, on large scales, by the ZA. Figs. 3 and 4 show the ratio of the correction terms to the "plane-parallel" limit (Eq. 9p, and compares the corrections in linear theory and the ZA. For the monopole and the quadropole our results are close to linear theory (presented in Castorina \& White 2018), but for $\ell=4$ we find a bigger difference on BAO scales with respect to the linear case.

A comparison of Figs. 3 and 4 shows the influence of the bias terms. Since we are treating the bias perturbatively, following Matsubara (2008), we expect this calculation to be most accurate at large scales. In linear theory and in plane-parallel approximation, biasing boils down to replacing $f \rightarrow f / b$ and $\xi_{\ell}^{(0)} \rightarrow b^{2} \xi_{\ell}^{(0)}$, which implies that bias suppresses redshift-space effects for $\ell=0$ and 2 but not for $\ell=4$. Figure 4 shows the ratio between the multipoles of the correlation function in linear theory and the ZA for a tracer with $b=1+b_{1}=2$ to the ones in the plane parallel limit. As expected, for $\ell=0$ the wide angle contributions are suppressed compared to the dark matter only case, but they are enhanced for $\ell=4$ where they can reach the $10 \%$ level near the acoustic scale $\left(s \sim 110 h^{-1} \mathrm{Mpc}\right)$.

It is clear from Eq. (36) that the wide-angle effects enter the bias terms differently than they do the matter terms. To further investigate the effect of biasing on wide angle redshift space distortions in the ZA, Fig. 5 compares the full result for halos and matter for $\ell=0,2,4$. As discussed above, in the plane-parallel approximation the hexadecapole of halos and matter is the same in linear theory and we therefore expect it to be more sensitive to wide angle effects. This is precisely what Fig. 5 shows.

Next we turn to the end-point approximation. Here we hold $s_{1}$ and $s$ fixed and integrate over $\mu_{1}$ to define the multipoles $\xi_{\ell}\left(s, s_{1}\right)$. This is no more difficult than the bisector case, numerically, since we can compute $\xi\left(\mathbf{s}_{1}, \mathbf{s}_{2}\right)$ with ease for any triangle configuration. However since this breaks the symmetry inherent in the bisector definition the corrections are larger. This is shown in Fig. 6 where we see the corrections becoming tens of percent at large scales. In the end-point approximation we also generate odd multipoles, comparable in size with $\ell=0,4$ multipoles above $s=120 h^{-1} \mathrm{Mpc}$ (Fig. 7). The odd terms result from our choice of coordinate system, and therefore are not real physical effects, and should not be confused with relativistic dipoles present in the cross-correlation between two different tracers (Bonvin et al. 2014 Iršič et al. 2016, Lepori et al. 2018). Nevertheless they should be taken into account in the search for GR effects. The trends seen in Figs. 6 and 7 can be explained by expanding our expressions in powers of $x$, as we shall do in the next section.

\subsection{Small angle expansion}

The second route is to expand the Zeldovich expression in Eq. (36) in powers of $x$ and look at the correction terms analytically in order to gauge their structure. This is useful since it allows us to better understand the differences with linear theory and in which limits the latter is recovered. Again let us begin with the expressions when $\hat{d}$ is taken to be the angle bisector. To this end, let us write

$$
\begin{aligned}
R_{2, i j} & =\left(1+f \hat{s}_{2} \hat{s}_{2}\right)_{i j} \\
& =\left(1+\frac{f}{s_{2}^{2}}[\mathbf{d}-t \mathbf{s}][\mathbf{d}-t \mathbf{s}]\right)_{i j} \\
& =(1+f \hat{d} \hat{d})_{i j}+f x\left[\mu \hat{d}_{i} \hat{d}_{j}-\frac{\hat{d}_{i} \hat{s}_{j}+\hat{d}_{j} \hat{s}_{i}}{2}\right] \\
& +\frac{f x^{2}}{4}\left[\hat{s}_{i} \hat{s}_{j}-\mu \hat{s}_{i} \hat{d}_{j}-\mu \hat{s}_{j} \hat{d}_{i}+\left(2 \mu^{2}-1\right) \hat{d}_{i} \hat{d}_{j}\right] \\
& +\mathcal{O}\left(x^{3}\right) \\
& \equiv \sum_{n} x^{n} R_{2, i j}^{(n)}
\end{aligned}
$$

where we have used $s_{2}^{2}=d^{2}-2 t d s \mu+t^{2} s^{2}=d^{2}(1-2 t x \mu+$ $\left.t^{2} x^{2}\right)$ and the expressions in Section 2 Similarly

$$
\begin{aligned}
R_{1, i j} & =\left(1+f \hat{s}_{1} \hat{s}_{1}\right)_{i j} \\
& =(1+f \hat{d} \hat{d})_{i j}-f x\left[\mu \hat{d}_{i} \hat{d}_{j}-\frac{\hat{d}_{i} \hat{s}_{j}+\hat{d}_{j} \hat{s}_{i}}{2}\right] \\
& +\frac{f x^{2}}{4}\left[\hat{s}_{i} \hat{s}_{j}-\mu \hat{s}_{i} \hat{d}_{j}-\mu \hat{s}_{j} \hat{d}_{i}+\left(2 \mu^{2}-1\right) \hat{d}_{i} \hat{d}_{j}\right] \\
& +\mathcal{O}\left(x^{3}\right)
\end{aligned}
$$

Note that $R_{i j}^{(1)} \equiv R_{1, i j}^{(1)}=-R_{2, i j}^{(1)}$ while $R_{1, i j}^{(2)}=R_{2, i j}^{(2)}$. Since $A_{i j}$ is symmetric, we see that the $\mathcal{O}(x)$ terms in $A_{s}$ vanish $^{4}$ (as we expect; Szalay et al. 1998, Reimberg et al. 2016 Castorina \& White 2018) and we are left with

$$
\begin{aligned}
A_{s, i j} & =A_{\infty, i j}+2 x^{2} \Sigma^{2}\left[+R_{i n}^{(1)} R_{n j}^{(1)}+2 R_{i n}^{(0)} R_{n j}^{(2)}\right] \\
& +2 x^{2}\left[-R_{i m}^{(1)} \xi_{m n} R_{n j}^{(1)}+2 R_{i m}^{(0)} \xi_{m n} R_{n j}^{(2)}\right]+\cdots
\end{aligned}
$$

where we have used $R_{i j}^{(n)}=R_{j i}^{(n)}$ for $n=0$ and 2. Note the $x^{2}$ correction only has support for $\mu^{0}, \mu^{1}$ and $\mu^{2}$, but this couples to the existing $\mu$-dependence of the $x^{0}$ terms to create a richer structure. Again it is important to note that in the $d^{3} q$ integral defining $1+\xi$ the values of $f, x, \mu, \hat{d}$ and $\hat{s}_{i}$ are constant.

To continue with the approximations, in order to gain some analytic intuition for the wide angle effects, let us follow Matsubara (2008) and in the decomposition $A_{i j}=$ $2\left[\Sigma^{2} \delta_{i j}-\xi_{i j}(q)\right]$, treat $\xi_{i j}$ as small. This is not optimal on smaller scales (Carlson et al. 2013), but will suffice to gain intuition on very large scales where the wide angle effects are largest. In the same spirit, we have also dropped the term in Eq. 36 going as $U_{i} U_{j}$, which is $\mathcal{O}\left(P_{L}^{2}\right)$.

Starting from our expression for the correlation function, Eq. (36), we can pull the $q$-independent piece of $\exp \left[-(1 / 2) k_{i} A_{s, i j} k_{j}\right]$ out of the integral. The plane-parallel term is

$$
\begin{aligned}
\mathcal{D}^{(0)} & =\exp \left[-\Sigma^{2} k_{i} R_{i m}^{(0)} R_{m j}^{(0)} k_{j}\right] \\
& =\exp \left[-k^{2} \Sigma^{2}\left(1+f[f+2] \mu_{k}^{2}\right)\right]
\end{aligned}
$$

\footnotetext{
${ }^{4}$ A similar cancellation of the $\mathcal{O}(x)$ terms occurs in $U_{s, i}$.
} 

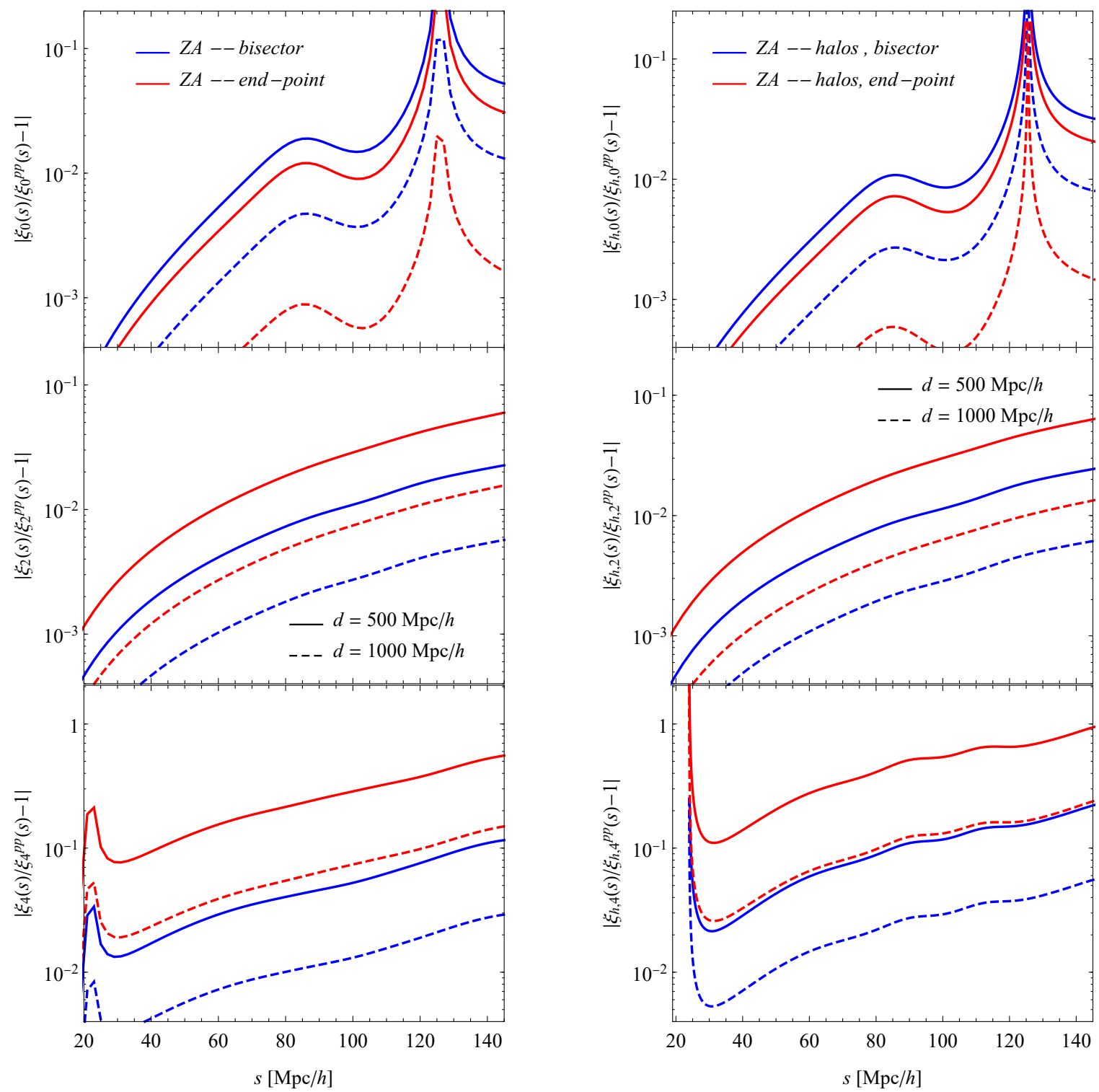

Figure 6. Same as Fig. 3 but now using the end-point approximation $\hat{d} \approx \hat{s}_{1}$ instead of the bisector. The left panels (for $\ell=0,2$ and 4 from top to bottom) show the matter predictions $(b=1)$ while the right panels show the halos with $b=2$.

where $\mu_{k}=\hat{k} \cdot \hat{d}$. This agrees with the form derived in Matsubara (2008) and can be rewritten as

$$
\mathcal{D}^{(0)}=\exp \left[-k_{\|}^{2} \Sigma_{\|}^{2}-k_{\perp}^{2} \Sigma_{\perp}^{2}\right]
$$

if $k_{\perp}^{2}=k^{2}\left(1-\mu_{k}^{2}\right), k_{\|}=k \mu_{k}, \Sigma_{\perp}=\Sigma$ and $\Sigma_{\|}=(1+f) \Sigma$. This term is responsible for the broadening of the BAO peak in the correlation function. The $\mathcal{O}\left(x^{2}\right)$ correction is

$$
1+x^{2} \mathcal{D}^{(2)}=\exp \left[-x^{2} \Sigma^{2} k_{i}\left(R_{i n}^{(1)} R_{n j}^{(1)}+2 R_{i n}^{(0)} R_{n j}^{(2)}\right) k_{j}+\cdots\right]
$$$$
\simeq 1+x^{2} k^{2} \Sigma^{2} \frac{1}{4} f(f+2)\left(\left(2 \mu^{2}-1\right) \mu_{k}^{2}-2 \mu \nu \mu_{k}+\nu^{2}\right)
$$

where we have defined $\nu=\hat{k} \cdot \hat{s}$. Note that this suggests that the broadening is opening angle dependent but that the correction is generally small.

Continue by expanding $\xi_{i j}(q)$ out of the exponential and doing the $d^{3} q$ integral. The lowest order term regains the usual expression (Kaiser 1987, Matsubara 2008)

$$
\begin{aligned}
\hat{k}_{i} R_{i j}^{(0)} \hat{k}_{j} \hat{k}_{m} R_{m n}^{(0)} \hat{k}_{n} P(k) & =\mathcal{K}^{(0)} P(k) \\
& =\left(1+f \mu_{k}^{2}\right)^{2} P(k)
\end{aligned}
$$

while the first correction for the matter is $x^{2} P$ times

$$
\begin{aligned}
\mathcal{K}^{(2)} & \equiv-\left(\hat{k}_{i} R_{i j}^{(1)} \hat{k}_{j}\right)^{2}+2 \hat{k}_{i} R_{i j}^{(0)} \hat{k}_{j} \hat{k}_{m} R_{m n}^{(2)} \hat{k}_{n} \\
& =-f^{2} \mu_{k}^{2}\left(\mu_{k} \mu-\nu\right)^{2} \\
& +\left(1+f \mu_{k}^{2}\right) \times \frac{f}{2}\left[\nu^{2}-2 \mu \mu_{k} \nu+\left(2 \mu^{2}-1\right) \mu_{k}^{2}\right] \\
& =\frac{f}{2}\left\{\frac{1}{3}\left(1-[1+f] \mu_{k}^{2}-3 f \mu_{k}^{4}\right)\right. \\
& -\mathcal{L}_{1}(\mu) \mathcal{L}_{1}(\nu) 2 \mu_{k}\left(1-f \mu_{k}^{2}\right) \\
& \left.+\mathcal{L}_{2}(\mu) \frac{4}{3} \mu_{k}^{2}+\mathcal{L}_{2}(\nu) \frac{2}{3}\left[1-f \mu_{k}^{2}\right]\right\}
\end{aligned}
$$




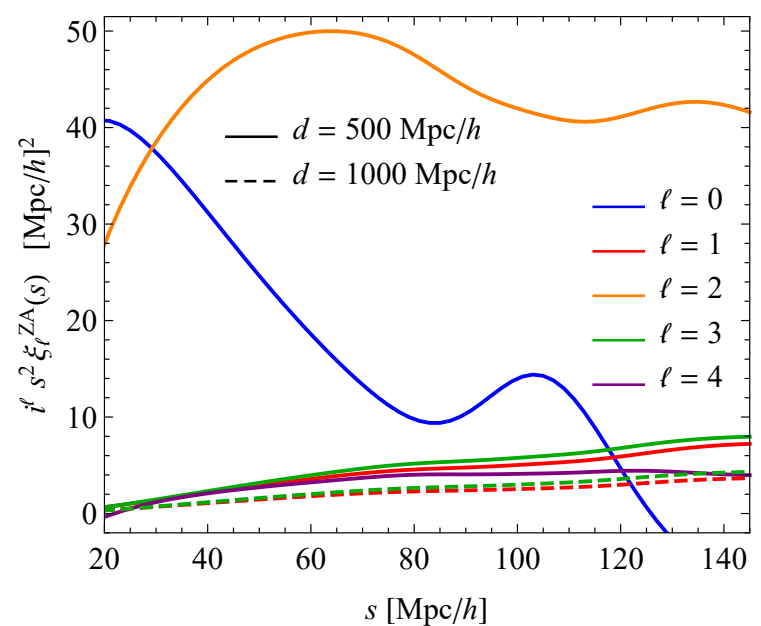

Figure 7. The dipole and octupole of the matter correlation function in the case of the end-point approximation for $s_{1}=$ $500 h^{-1} \mathrm{Mpc}$ and $1 h^{-1} \mathrm{Gpc}$, compared to the the even multipoles in the flat-sky limit.

The bias terms are enumerated in Appendix B It is however important to note that the leading wide angle correction to the bias terms, Eq. (B4), is different than the one described above as it does not receive a contribution $\propto\left(k_{i} R_{i j}^{(1)} k_{j}\right)^{2}$. Our expression, for the matter, is thus

$$
\begin{aligned}
\xi^{(s)}(s, d, \mu) & =\int \frac{\mathrm{d}^{3} k}{(2 \pi)^{3}} e^{i \mathbf{k} \cdot \mathbf{s}} \mathcal{D}^{(0)}\left(k, \mu_{k}\right) \\
& \times P(k)\left[\mathcal{K}^{(0)}+x^{2} \mathcal{K}^{(2)}\right]\left(1+x^{2} \mathcal{D}^{(2)}\right)
\end{aligned}
$$

The $\mathcal{O}\left(x^{0}\right)$ term is simply the normal Kaiser expression with $P \rightarrow \mathcal{D}^{(0)} P$. The $\mathcal{O}\left(x^{2}\right)$ terms give the leading wide angle correction.

Before we study the $\mathcal{O}\left(x^{2}\right)$ terms let us quickly review the calculation to lowest order:

$$
\begin{aligned}
\xi_{\ell} & \ni(2 \ell+1) \int \frac{\mathrm{d}^{3} k}{(2 \pi)^{3}} \frac{\mathrm{d}^{2} \hat{s}}{4 \pi} e^{i \mathbf{k} \cdot \mathbf{s}} \mathcal{L}_{\ell}(\hat{s} \cdot \hat{d}) \\
& \times \mathcal{D}^{(0)}\left(k, \mu_{k}\right)\left(1+f \mu_{k}^{2}\right)^{2} P(k) \\
& =(2 \ell+1) i^{\ell} \int \frac{k^{2} \mathrm{~d} k}{2 \pi^{2}} P(k) j_{\ell}(k s) \\
& \times \int \frac{\mathrm{d} \Omega_{k}}{4 \pi} \mathcal{L}_{\ell}\left(\mu_{k}\right) \mathcal{D}^{(0)}\left(k, \mu_{k}\right)\left(1+f \mu_{k}^{2}\right)^{2}
\end{aligned}
$$

where $\mu_{k}=\hat{k} \cdot \hat{d}$. In the absence of the damping term, the $d \Omega_{k}$ integral gives the usual multipoles $\ell=0,2$ and 4 and one recovers Eq. 9. The anisotropic damping also populates $\ell>4$. While the results of the $d \Omega_{k}$ integral can be written in closed form, they are not illuminating and so will be omitted (see e.g. Peacock \& Dodds 1994, for further discussion).

Now we consider the $\mathcal{O}\left(x^{2}\right)$ terms. For these terms $\xi_{\ell}$ has $\hat{s}$ dependence not just through $\exp [i \mathbf{k} \cdot \mathbf{s}]$ and $\mathcal{L}_{\ell}(\hat{s} \cdot \hat{d})$ but also through the $\mu$ and $\nu$ terms in Eq. (57). However the terms are at most quadratic in these variables. The $\mathcal{O}\left(x^{2}\right)$ contribution to $\xi_{\ell}$ is

$$
\begin{aligned}
\xi_{\ell} & \ni(2 \ell+1) x^{2} \int \frac{\mathrm{d}^{3} k}{(2 \pi)^{3}} \frac{\mathrm{d} \hat{s}}{4 \pi} e^{i \mathbf{k} \cdot \mathbf{s}} \mathcal{L}_{\ell}(\hat{s} \cdot \hat{d}) \\
& \times \mathcal{D}^{(0)}\left(k, \mu_{k}\right) P(k)\left\{\mathcal{K}^{(0)} \mathcal{D}^{(2)}+\mathcal{K}^{(2)}\right\}
\end{aligned}
$$

Upon performing the integral over $\mathrm{d}^{2} \hat{s}$ using the expressions in Appendix $C$ the contribution is of the form

$$
\begin{aligned}
\xi_{\ell} & \ni(2 \ell+1) x^{2} \int \frac{k^{2} \mathrm{~d} k}{2 \pi^{2}} P(k) \sum_{L} i^{L} j_{L}(k s) \\
& \times \int \frac{\mathrm{d} \Omega_{k}}{4 \pi} \mathcal{C}_{L}^{\ell}\left(\mu_{k}\right) \mathcal{D}^{(0)}\left(k, \mu_{k}\right)
\end{aligned}
$$

where the $\mathcal{C}_{L}^{\ell}$ are polynomials in $\mu_{k}$. We give the general expressions for $\mathcal{C}_{L}^{\ell}$ in Appendix $\mathrm{C}$. As an example

$$
\begin{aligned}
& C_{0}^{0}=\frac{1}{12} f(f+2) k^{2} \Sigma^{2}\left(1-3 \mu_{k}^{2}\right)\left(f \mu_{k}^{2}+1\right)^{2} \\
& -\frac{1}{6} f\left(f \mu_{k}^{4}+(f+3) \mu_{k}^{2}-1\right)
\end{aligned}
$$

The polynomials for high $\ell$ are long and we shall not reproduce them here. Similar to the plane parallel limit in Eq. (59) the angular integral in Eq. (61) can be evaluated analytically, but the resulting expression is not very illuminating. The important point to note is that in general $\xi_{\ell}$ now contains contributions $j_{L}(k s)$ for $L \neq \ell$, as was the case for linear theory (see the discussion in Castorina \& White 2018). In fact, in the limit $\mathcal{D}^{(2)} \rightarrow 0$ and $\mathcal{D}^{(0)} \rightarrow 1$ we regain the earlier, linear theory, results. This can be seen as an alternative route to those results, first derived by Szalay et al. (1998).

It is straightforward to convert thes $€^{5}$ bisector-based results to the case where $\hat{d}$ is approximated by $\hat{s}_{1}$, i.e. the end-point approximation. The lowest order terms (Eq. 59p are unchanged and for the terms in Eq. 61, which are already $\mathcal{O}\left(x^{2}\right)$, we can simply replace $d$ with $s_{1}$ (i.e. $x \rightarrow x_{1}$ ). The change $\mu \rightarrow \mu_{1}$ mixes multipoles since

$$
\mathcal{L}_{\ell}(\mu)=\mathcal{L}_{\ell}\left(\mu_{1}\right)+\frac{x_{1}}{2} \sqrt{1-\mu_{1}^{2}} \mathcal{L}_{\ell}^{1}\left(\mu_{1}\right)-\frac{x_{1}^{2}}{8}\left(1-\mu_{1}^{2}\right) \mathcal{L}_{\ell}^{2}\left(\mu_{1}\right)
$$

through $\mathcal{O}\left(x_{1}^{2}\right)$. This populates the odd $\ell$. Thus to the wideangle bisector terms we must add the terms in Table 1 times $\xi_{L}^{p p}$. Denoting the end-point expansion with a tilde, as for $\tilde{P}_{\ell}^{(n)}(k)$, we have for example $\tilde{\xi}_{0}(s)=\xi_{0}(s)+\left(x_{1}^{2} / 5\right) \xi_{2}^{p p}(s)$ with $\xi_{0}(s)$ the bisector expression including the $\mathcal{O}\left(x^{2}\right)$ terms and $\xi_{2}^{p p}$ the plane-parallel limit. Since $\xi_{2}^{p p}<0$, the formulae above explain why in Figure 6 the end-point monopole is less affected by wide angle effects than the bisectordefine monopole. Table 1 explicitly shows that odd multipoles do not carry any other extra-information, as they are proportional to the multipoles in the plane-parallel limit, e.g. $\tilde{\xi}_{1}\left(s, s_{1}\right)=-3 / 5 x_{1} \xi_{2}^{p p}(s)$.

\subsection{Power spectrum}

We can express the expectation value of the Yamamoto estimator for the power spectrum as an integral over $\xi(s, d, \mu)$ and use Eq. (57) to study the impact of the wide angle terms,

5 The conversions also hold for the linear theory results presented in Castorina \& White (2018). 


\begin{tabular}{ccc}
$\ell$ & $L=2$ & $L=4$ \\
\hline 0 & $x_{1}^{2} / 5$ & 0 \\
1 & $-3 x_{1} / 5$ & 0 \\
2 & $-2 x_{1}^{2} / 7$ & $5 x_{1}^{2} / 7$ \\
3 & $3 x_{1} / 5$ & $-10 x_{1} / 9$ \\
4 & $3 x_{1}^{2} / 35$ & $-90 x_{1}^{2} / 77$
\end{tabular}

Table 1. The coefficients of the additional contributions to $\xi_{\ell}$ which are generated by the use of the end-point approximation $\left(\hat{d} \simeq \hat{s}_{1}\right)$ as described in the text.

on large scales and to $\mathcal{O}\left(x^{2}\right)$. Using $\hat{d}$ as the line of sight, the Yamamoto estimator is simply the Hankel transform of our correlation function multipoles:

$$
\begin{aligned}
\left\langle P_{L}^{Y}(k)\right\rangle & =(2 L+1) \int \frac{\mathrm{d} \Omega_{\mathbf{k}}}{4 \pi} \frac{\mathrm{d}^{3} d}{V} \mathrm{~d}^{3} s e^{-i \mathbf{k} \cdot \mathbf{s}} \mathcal{L}_{L}(\hat{k} \cdot \hat{d}) \xi(s, d, \mu) \\
& =(2 L+1) \int \frac{\mathrm{d} \Omega_{\mathbf{k}}}{4 \pi} \frac{\mathrm{d}^{3} d}{V} \mathrm{~d}^{3} s e^{-i \mathbf{k} \cdot \mathbf{s}} \mathcal{L}_{L}(\hat{k} \cdot \hat{d}) \\
& \times \sum_{\ell} \xi_{\ell}(s, d) \mathcal{L}_{\ell}(\hat{s} \cdot \hat{d}) \\
& =(4 \pi)(-i)^{L} \int \frac{\mathrm{d}^{3} d}{V} s^{2} \mathrm{~d} s j_{L}(k s) \xi_{L}(s, d)
\end{aligned}
$$

as in Eq. (14). The lowest order terms simplify upon using the completeness relation

$$
\int s^{2} \mathrm{~d} s j_{\ell}(k s) j_{\ell}\left(k^{\prime} s\right)=\frac{\pi}{2 k k^{\prime}} \delta^{(D)}\left(k-k^{\prime}\right) .
$$

The $\mathrm{d} s$ integral times the Bessel function "undoes" the $\mathrm{d} k$ integral times the Bessel function in Eq. (59) and

$$
\begin{aligned}
\left\langle P_{L}^{Y,(0)}(k)\right\rangle & =(2 L+1) P(k) \\
& \times \int \frac{\mathrm{d} \Omega_{k}}{4 \pi} \mathcal{D}^{(0)}\left(k, \mu_{k}\right) \mathcal{L}_{L}\left(\mu_{k}\right)\left(1+f \mu_{k}^{2}\right)^{2}
\end{aligned}
$$

which recovers the expression in Matsubara (2008). Note the well-known exponential damping of the Zeldovich power spectrum. Additional high- $k$ power is generated by contributions which are isolated to small $r$ in configuration space. Various models for this missing power have been proposed, either heuristic (Eisenstein et al.|2007||Seo et al.|2008), based on the halo model (Mohammed \& Seljak 2014| Seljak \& Vlah 2015) or on effective field theory (Porto et al. 2014, Vlah et al. 2015, 2016).

At second order plugging Eq. (61) into Eq. (14) does not further simplify since the integral of $j$ 's of different orders does not vanish. We are thus left with

$$
\begin{aligned}
\left\langle P_{L}^{Y}(k)\right\rangle & \ni(2 L+1) x^{2} \int \frac{\mathrm{d}^{3} d}{V} \frac{\mathrm{d}^{3} k^{\prime}}{(2 \pi)^{3}} P\left(k^{\prime}\right) \mathcal{D}^{(0)}\left(k^{\prime}, \mu_{k}^{\prime}\right) \\
& \times \sum_{J} C_{J}^{L}\left(\mu_{k}^{\prime}\right)(4 \pi) \int s^{2} \mathrm{~d} s j_{L}(k s) j_{J}\left(k^{\prime} s\right)
\end{aligned}
$$

The last integral, over $\mathrm{d} s$, can be expressed analytically using hypergeometric functions, but the final expression does not provide any further insights.

The fast FFT estimator in Eq. 200 can be expressed in a similar form using either the bisector or the end-point as the LOS. The former has been presented in Eq. 32 of (Castorina \& White 2018), while the latter can be obtained from Eqs. 30-31 of (Castorina \& White 2018) using the mapping in Eq. (64) and Table 1]

\section{CONCLUSIONS}

The physics of electromagnetic emission from moving objects, which imprints a contribution from the line-of-sight peculiar velocity onto the observed redshift of extragalactic objects, breaks the translational invariance of our theories down to a rotational symmetry. The induced effects, which become important in 2-point clustering statistics when the opening angle between the two points becomes appreciable, go under the name of "wide angle effects". Since these effects are largest on large scales, most earlier papers have assumed Eulerian, linear perturbation theory in their analyses. In this paper we have shown that wide angle effects can be easily handled within the context of Lagrangian perturbation theory, allowing an efficient resummation of the linear displacements which is particularly important for modeling BAO.

Beyond the plane-parallel approximation the two point function is most easily expressed in terms of the correlation function or the multi-frequency angular power spectrum (MAPS). We developed the relationship between these probes and showed how the MAPS can be computed beyond linear theory. We investigated the relationship of these statistics to the multipole moments of the power spectrum computed with the Yamamoto estimator, using either the bisector or end-point conventions for the line of sight direction.

We have compared our calculation, numerically and analytically, to the earlier linear theory calculations. Except near the BAO peak, where linear theory does a poor job, the size of the corrections for the $\ell=0$ and 2 multipole moments of the correlation function are very similar in the $\mathrm{ZA}$ and in linear theory. For $\ell=4$ the corrections predicted in the ZA are larger than the linear theory predictions. The corrections are significantly larger if the end-point convention is used to define the line of sight than if the bisector approximation is made.

We note that it is relatively straightforward, if tedious, to extend our analysis to higher order in (Lagrangian) perturbation theory. This would allow a comparison of the size of the wide angle terms to those from second order dynamics. What is much more difficult is an extension of this work to schemes such as the streaming model (e.g. Vlah et al. 2016, and references therein), in which a fixed line of sight is critical to the simplification of the final expressions. However, on small scales the wide-angle terms are small while on large scales the corrections to the dynamics are small. This suggests a perturbative approach where the wide-angle corrections are computed at low order (as we have done here) and used to correct the more sophisticated model, that is computed in the plane-parallel approximation.

M.W. is supported by the U.S. Department of Energy and by NSF grant number 1713791. This work made extensive use of the NASA Astrophysics Data System and of the astro-ph preprint archive at arXiv.org. 


\section{APPENDIX A: FLAT SKY APPROXIMATION TO THE MAPS}

In the main text we discussed the relationship of the MAPS, $C_{\ell}\left(s_{1}, s_{2}\right)$, to the correlation function and power spectrum. If we make the small-angle, or flat-sky, approximation and define $s_{\|}=s \mu$ and $s_{\perp}=s \sqrt{1-\mu^{2}}$ then

$$
\begin{aligned}
C_{\ell}\left(s_{1}, s_{2}\right) & =2 \pi \int \mathrm{d}(\cos \theta) \xi(s, d, \mu) \mathcal{L}_{\ell}(\cos \theta) \\
& \simeq 2 \pi \int \widetilde{\omega} \mathrm{d} \widetilde{\omega} \xi\left(s_{\perp}, s_{\|}, d\right) J_{0}(\ell \widetilde{\omega})
\end{aligned}
$$

where $\widetilde{\omega}=2 \sin (\theta / 2) \simeq \theta$. Changing arguments to $s_{1}-s_{2} \simeq$ $s_{\|}+\mathcal{O}\left(x^{3}\right)$ and $\frac{1}{2}\left(s_{1}+s_{2}\right) \simeq d+\mathcal{O}\left(x^{2}\right)$ and writing $\ell=k_{\perp} d$ so $\ell \widetilde{\omega} \simeq k_{\perp} s_{\perp}$ we find

$$
C_{\ell}\left(s_{\|}, d\right) \simeq \int \frac{\mathrm{d}^{2} s_{\perp}}{d^{2}} \xi\left(s_{\|}, s_{\perp}, d\right) e^{i \mathbf{k}_{\perp} \cdot \mathbf{s}_{\perp}}
$$

where we have used the Rayleigh expansion of the planewave in cylindrical coordinates and the azimuthal symmetry of the integral. Thus the MAPS, in the flat-sky limit, is the 2D Fourier transform of the correlation function. A further Fourier transform (in $s_{\|}$) returns $P(\mathbf{k})$. Alternatively the MAPS is the line-of-sight Fourier transform of $P\left(k_{\|}, k_{\perp}\right)$ :

$$
C_{\ell}\left(s_{\|}, d\right) \simeq \int_{0}^{\infty} \frac{d k_{\|}}{\pi d^{2}} P\left(k_{\perp}=\ell / d, k_{\|}\right) \cos \left(k_{\|} s_{\|}\right)
$$

For an alternative derivation, at the level of the fields, see the appendices of White et al. (1999), Datta et al. (2007) or White \& Padmanabhan (2017).

\section{APPENDIX B: BIAS TERMS}

The low- $k$ expansion of the bias terms in Eq. 36 follows very similar steps to the one for the matter terms presented in the main text. The $b_{1}^{2}$ term does not carry any extra redshift space dependence and therefore is identical to the expansion of $\xi_{i j}$ in Eq. (54). The $b_{1}$ piece is also straightforward. Expanding $U_{s, i}$ to quadratic order in $x$ we get

$$
U_{s, i}(q)=R_{i j}^{(0)} U_{j}(q)+R_{i j}^{(2)} U_{j}(q) \equiv U_{s, i}^{(0)}(q)+U_{s, i}^{(2)}(q)
$$

which we can then plug back into Eq. (36). At lowest order,

$$
\begin{aligned}
\xi_{b_{1}}^{(0)}(s, d, \mu) & =2 b_{1} \int \frac{\mathrm{d}^{3} k}{(2 \pi)^{3}} e^{i \mathbf{q} \cdot \mathbf{s}} \mathcal{D}^{(0)} \int \mathrm{d}^{3} q \int \frac{\mathrm{d}^{3} p}{(2 \pi)^{3}} e^{i \mathbf{q} \cdot(\mathbf{p}-\mathbf{k})} \\
& \times k_{i}\left(\delta_{i j}+f \hat{d}_{i} \hat{d}_{j}\right) i \frac{p_{j}}{p^{2}} P_{L}(p) \\
& =2 b_{1} \int \frac{\mathrm{d}^{3} k}{(2 \pi)^{3}} \mathcal{D}^{(0)} e^{i \mathbf{q} \cdot \mathbf{s}}\left(1+f \mu_{k}^{2}\right) P_{L}(k)
\end{aligned}
$$

and we recover the familiar Kaiser result

$$
P_{L, s}\left(k, \mu_{k}\right)=\left(\left[1+b_{1}\right]+f \mu_{k}^{2}\right)^{2} P_{L}(k)
$$

upon recalling the large scale (Eulerian) bias is $b=1+b_{1}$. At second order

$$
\begin{aligned}
\xi_{b_{1}}^{(2)}(s, d, \mu) & =2 b_{1} x^{2} \int \frac{\mathrm{d}^{3} k}{(2 \pi)^{3}} e^{i \mathbf{q} \cdot \mathbf{s}} \int \mathrm{d}^{3} q \int \frac{\mathrm{d}^{3} p}{(2 \pi)^{3}} e^{i \mathbf{q} \cdot(\mathbf{p}-\mathbf{k})} \\
& \times \mathcal{D}^{(0)} k_{i}\left(R_{i j}^{(2)}+\mathcal{D}^{(2)} R_{i j}^{(0)}\right) i \frac{p_{j}}{p^{2}} P_{L}(p) \\
& =2 b_{1} x^{2} \int \frac{\mathrm{d}^{3} k}{(2 \pi)^{3}} e^{i \mathbf{k} \cdot \mathbf{s}} P_{L}(k) \mathcal{D}^{(0)} \\
& \times\left[\frac{f}{4}\left(\nu^{2}-2 \mu \mu_{k} \nu+\left(2 \mu^{2}-1\right) \mu_{k}^{2}\right)\right. \\
& \left.+\mathcal{D}^{(2)}\left(1+f \mu_{k}^{2}\right)\right]
\end{aligned}
$$

It is worth pointing out that leading order wide angle contribution calculated above differs from the dark matter one in Eq. 55 even neglecting the expansion of the damping term. This is a new feature of the ZA and it explains why the $\mathrm{ZA}$ is more different from linear theory for halos than it is for dark matter.

\section{APPENDIX C: THE WIDE ANGLE TERMS}

As described in the main text, for the $\mathcal{O}\left(x^{2}\right)$ terms, $\xi_{\ell}$ has $\hat{s}$ dependence not just through $\exp [i \mathbf{k} \cdot \mathbf{s}]$ and $\mathcal{L}_{\ell}(\hat{s} \cdot \hat{d})$ but also through the $\mu$ and $\nu$ terms in Eq. (57). However these are at most quadratic in these variables. We rewrite each of the terms using e.g. $\nu^{2}=(1 / 3)+(2 / 3) \mathcal{L}_{2}(\nu)$ and $\mu \nu=$ $\mathcal{L}_{1}(\mu) \mathcal{L}_{1}(\nu)$.

The $\mathcal{O}\left(x^{2}\right)$ terms which are independent of $\mu$ and $\nu$ go through as for the $\mathcal{O}\left(x^{0}\right)$ terms, giving a Hankel transform of order $\ell$ :

$$
\int \frac{\mathrm{d} \hat{s}}{4 \pi} e^{i \mathbf{k} \cdot \mathbf{s}} \mathcal{L}_{\ell}(\hat{s} \cdot \hat{d})=i^{\ell} j_{\ell}(k s) \mathcal{L}_{\ell}(\hat{k} \cdot \hat{d})
$$

The other terms are proportional to $\mathcal{L}_{2}(\nu), \mathcal{L}_{2}(\mu)$ and $\mathcal{L}_{1}(\nu) \mathcal{L}_{1}(\mu)$ and will additionally give Hankel transforms of different orders: $L \neq \ell$. Using the angular momentum addition theorem for Legendre polynomials

$$
\mathcal{L}_{\ell_{1}}(\mu) \mathcal{L}_{\ell_{2}}(\mu)=\sum_{L}\left(\begin{array}{ccc}
\ell_{1} & \ell_{2} & L \\
0 & 0 & 0
\end{array}\right)^{2}(2 L+1) \mathcal{L}_{L}(\mu)
$$

the Rayleigh expansion of a plane wave

$$
e^{i \mathbf{k} \cdot \mathbf{s}}=\sum_{\ell} i^{\ell}(2 \ell+1) j_{\ell}(k s) \mathcal{L}_{\ell}(\hat{k} \cdot \hat{s})
$$

and the addition theorem

$$
\mathcal{L}_{\ell}\left(\hat{n}_{1} \cdot \hat{n}_{2}\right)=\frac{4 \pi}{2 \ell+1} \sum_{m} Y_{\ell m}\left(\hat{n}_{1}\right) Y_{\ell m}^{\star}\left(\hat{n}_{2}\right)
$$

one can show

$$
\begin{aligned}
& \int \frac{\mathrm{d} \hat{s}}{4 \pi} e^{i \mathbf{k} \cdot \mathbf{s}} \mathcal{L}_{\ell}(\hat{s} \cdot \hat{d}) \mathcal{L}_{2}(\hat{s} \cdot \hat{k}) \\
& =\sum_{L}\left(\begin{array}{lll}
L & 2 & \ell \\
0 & 0 & 0
\end{array}\right)^{2}(2 L+1) i^{L} j_{L}(k s) \mathcal{L}_{\ell}(\hat{k} \cdot \hat{d})
\end{aligned}
$$

For each $\ell$ only a finite number of terms with $L \neq \ell$ contribute and $L$ is even. We also have

$$
\begin{aligned}
& \int \frac{\mathrm{d} \hat{s}}{4 \pi} e^{i \mathbf{k} \cdot \mathbf{s}} \mathcal{L}_{\ell}(\hat{s} \cdot \hat{d}) \mathcal{L}_{2}(\hat{s} \cdot \hat{d}) \\
& =\sum_{L}\left(\begin{array}{lll}
L & 2 & \ell \\
0 & 0 & 0
\end{array}\right)^{2}(2 L+1) i^{L} j_{L}(k s) \mathcal{L}_{L}(\hat{k} \cdot \hat{d})
\end{aligned}
$$


which also contains only even $L$. Finally

$$
\begin{aligned}
& \int \frac{\mathrm{d} \hat{s}}{4 \pi} e^{i \mathbf{k} \cdot \mathbf{s}} \mathcal{L}_{\ell}(\hat{s} \cdot \hat{d}) \mathcal{L}_{1}(\hat{s} \cdot \hat{k}) \mathcal{L}_{1}(\hat{s} \cdot \hat{d}) \\
& =\sum_{J L}\left(\begin{array}{lll}
\ell & 1 & J \\
0 & 0 & 0
\end{array}\right)^{2}\left(\begin{array}{lll}
L & 1 & J \\
0 & 0 & 0
\end{array}\right)^{2}(2 L+1)(2 J+1) i^{L} \\
& \times j_{L}(k s) \mathcal{L}_{J}(\hat{k} \cdot \hat{d})
\end{aligned}
$$

The double sum is also finite and contains only even $L$ 's. The $\mathcal{O}\left(x^{2}\right)$ contribution to $\xi_{\ell}$ is then

$$
\begin{aligned}
& (2 \ell+1) x^{2} \int \frac{\mathrm{d}^{3} k}{(2 \pi)^{3}} \frac{\mathrm{d} \hat{s}}{4 \pi} e^{i \mathbf{k} \cdot \mathbf{s}} \mathcal{L}_{\ell}(\mu) \mathcal{D}^{(0)} P(k) \\
& \left\{T_{00}+T_{11} \mathcal{L}_{1}(\mu) \mathcal{L}_{1}(\nu)+T_{20} \mathcal{L}_{2}(\mu)+T_{02} \mathcal{L}_{2}(\nu)\right\} \quad(\mathrm{C} 8) \\
& =x^{2} \int \frac{\mathrm{d}^{3} k}{(2 \pi)^{3}} \mathcal{D}^{(0)}\left(k, \mu_{k}\right) P(k) \sum_{L} i^{L} j_{L}(k s) \\
& \times(2 \ell+1)\left\{T_{00} \delta_{L \ell} \mathcal{L}_{\ell}\left(\mu_{k}\right)\right. \\
& +T_{11} \sum_{J}(2 L+1)(2 J+1)\left(\begin{array}{lll}
L & 1 & J \\
0 & 0 & 0
\end{array}\right)^{2}\left(\begin{array}{lll}
\ell & 1 & J \\
0 & 0 & 0
\end{array}\right)^{2} \mathcal{L}_{J}\left(\mu_{k}\right) \\
& +T_{20}(2 L+1)\left(\begin{array}{lll}
\ell & 2 & L \\
0 & 0 & 0
\end{array}\right)^{2} \mathcal{L}_{L}\left(\mu_{k}\right) \\
& \left.+T_{02}(2 L+1)\left(\begin{array}{lll}
L & 2 & \ell \\
0 & 0 & 0
\end{array}\right)^{2} \mathcal{L}_{\ell}\left(\mu_{k}\right)\right\}
\end{aligned}
$$

where

$$
\begin{aligned}
& T_{00}=\frac{1}{12} f\left((f+2) k^{2} \Sigma^{2}\left(\mu_{k}^{2}-1\right)\left(f \mu_{k}^{2}+1\right)^{2}\right. \\
& \left.-6 f \mu_{k}^{4}-2(f+1) \mu_{k}^{2}+2\right) \\
& T_{11}=\frac{1}{2} f \mu_{k}\left(-(f+2) k^{2} \Sigma^{2}\left(f \mu_{k}^{2}+1\right)^{2}+2 f \mu_{k}^{2}-2\right) \\
& T_{20}=\frac{1}{3} f\left((f+2) k^{2} \Sigma^{2}\left(f \mu_{k}^{3}+\mu_{k}\right)^{2}+2 \mu_{k}^{2}\right) \\
& T_{02}=\frac{1}{6}\left(f(f+2) k^{2} \Sigma^{2}\left(f \mu_{k}^{2}+1\right)^{2}-2 f\left(f \mu_{k}^{2}-1\right)\right)
\end{aligned}
$$

The triangle condition on the $3 j$ symbols ensures that only a finite number of terms contribute for any $\ell$, and it is straightforward to compute $\mathcal{C}_{L}^{\ell}$ of the main text from the above expressions. The symmetry of the problem ensures that the dipole terms which one might naively think appear in the sum in fact cancel exactly.

\section{REFERENCES}

Bianchi D., Gil-Marín H., Ruggeri R., Percival W. J., 2015, Mon. Not. R. Astron. Soc. 453, L11

Bonvin C., Durrer R., 2011, Phys. Rev. D, 84, 063505

Bonvin C., Hui L., Gaztañaga E., 2014, Phys. Rev. D, 89, 083535

Carlson J., Reid B., White M., 2013, Mon. Not. R. Astron. Soc. 429, 1674

Castorina E., White M., 2018, Mon. Not. R. Astron. Soc. 476,4403

Chuang C.-H., Kitaura F.-S., Prada F., Zhao C., Yepes G., 2015, Mon. Not. R. Astron. Soc. 446, 2621
Coles P., Melott A. L., Shandarin S. F., 1993, Mon. Not. R. Astron. Soc. 260, 765

Datta K. K., Choudhury T. R., Bharadwaj S., 2007, Mon. Not. R. Astron. Soc. 378, 119

Dodelson S., 2003, Modern cosmology

Doroshkevich A. G., Kotok E. V., Poliudov A. N., Shandarin S. F., Sigov I. S., Novikov I. D., 1980, Mon. Not. R. Astron. Soc. 192, 321

Eisenstein D. J., Seo H.-J., White M., 2007, Astrophys. J. 664,660

Fisher K. B., Scharf C. A., Lahav O., 1994, Mon. Not. R. Astron. Soc. 266, 219

Guzzo L., et al., 2008, Nature, 451, 541

Hahn C., Scoccimarro R., Blanton M. R., Tinker J. L., Rodríguez-Torres S. A., 2017, Mon. Not. R. Astron. Soc. 467, 1940

Hamilton A. J. S., 1992, Astrophys. J. Lett., 385, L5

Hamilton A. J. S., 1998, in Hamilton D., ed., Astrophysics and Space Science Library Vol. 231, The Evolving Universe. p. 185 (arXiv:astro-ph/9708102), doi:10.1007/978-94-011-4960-0¹7

Hamilton A. J. S., Culhane M., 1996a, Mon. Not. R. Astron. Soc. 278, 73

Hamilton A. J. S., Culhane M., 1996b, Mon. Not. R. Astron. Soc. 278, 73

Hand N., Li Y., Slepian Z., Seljak U., 2017, Journal of Cosmology and Astro-Particle Physics, 7, 002

Heavens A. F., Taylor A. N., 1995, Mon. Not. R. Astron. Soc. 275,483

Hidding J., Shandarin S. F., van de Weygaert R., 2014, Mon. Not. R. Astron. Soc., 437, 3442

Iršič V., Di Dio E., Viel M., 2016, Journal of Cosmology and Astro-Particle Physics, 2, 051

Joyce A., Jain B., Khoury J., Trodden M., 2015, Phys. Rep. 568,1

Kaiser N., 1987, Mon. Not. R. Astron. Soc. 227, 1

Lahav O., Fisher K. B., Hoffman Y., Scharf C. A., Zaroubi S., 1994, Astrophys. J. Lett. 423, L93

Lepori F., Di Dio E., Villa E., Viel M., 2018, Journal of Cosmology and Astro-Particle Physics, 5, 043

Lesgourgues J., Pastor S., 2006, Phys. Rep., 429, 307

Liu A., Zhang Y., Parsons A. R., 2016, Astrophys. J. 833, 242

Matsubara T., 2008, Phys. Rev. D , 78, 083519

McCullagh N., Szalay A. S., 2012, Astrophys. J. 752, 21

Mohammed I., Seljak U., 2014, Mon. Not. R. Astron. Soc. 445,3382

Noh Y., White M., Padmanabhan N., 2009, Phys. Rev. D 80,123501

Padmanabhan N., Tegmark M., Hamilton A. J. S., 2001, Astrophys. J. 550,52

Padmanabhan N., White M., Cohn J. D., 2009, Phys. Rev. D , 79, 063523

Pápai P., Szapudi I., 2008, Mon. Not. R. Astron. Soc. ,389, 292

Passaglia S., Manzotti A., Dodelson S., 2017, Phys. Rev. D I. 95,123508

Pauls J. L., Melott A. L., 1995, Mon. Not. R. Astron. Soc. П. 274,99

Peacock J. A., 1999, Cosmological Physics

Peacock J. A., Dodds S. J., 1994, Mon. Not. R. Astron. Soc. |. 267,1020 
Percival W. J., White M., 2009, Mon. Not. R. Astron. Soc. [, 393,297

Percival W. J., et al., 2004, Mon. Not. R. Astron. Soc. 353, 1201

Porto R. A., Senatore L., Zaldarriaga M., 2014, Journal of Cosmology and Astro-Particle Physics, 5, 022

Pratten G., Munshi D., 2013, Mon. Not. R. Astron. Soc. 436,3792

Raccanelli A., Bertacca D., Doré O., Maartens R., 2014, Journal of Cosmology and Astro-Particle Physics, 8, 022

Reimberg P., Bernardeau F., Pitrou C., 2016, Journal of Cosmology and Astro-Particle Physics, 1, 048

Sahni V., Coles P., 1995, Phys. Rep., 262, 1

Scoccimarro R., 2015, Phys. Rev. D 92, 083532

Seljak U., Vlah Z., 2015, Phys. Rev. D , 91, 123516

Seo H.-J., Siegel E. R., Eisenstein D. J., White M., 2008, Astrophys. J. 686, 13

Shaw J. R., Lewis A., 2008, Phys. Rev. D, 78, 103512

Shaw J. R., Sigurdson K., Pen U.-L., Stebbins A., Sitwell M., 2014, Astrophys. J. 781, 57

Slepian Z., Eisenstein D. J., 2015, preprint, (arXiv:1510.04809)

Szalay A. S., Matsubara T., Landy S. D., 1998, Astrophys. J. Lett. 498, L1

Szapudi I., 2004, Astrophys. J. , 614, 51

Tassev S., 2014a, Journal of Cosmology and Astro-Particle Physics, 6, 008

Tassev S., 2014b, Journal of Cosmology and Astro-Particle Physics, 6, 012

Tassev S., Zaldarriaga M., 2012a, Journal of Cosmology and Astro-Particle Physics, 4, 013

Tassev S., Zaldarriaga M., 2012b, Journal of Cosmology and Astro-Particle Physics, 10, 006

Vlah Z., White M., Aviles A., 2015, Journal of Cosmology and Astro-Particle Physics, 9, 014

Vlah Z., Castorina E., White M., 2016, Journal of Cosmology and Astro-Particle Physics, 12, 007

Watson G. N., 1966, A Treatise on the Theory of Bessel Functions, 2nd ed

Weinberg D. H., Mortonson M. J., Eisenstein D. J., Hirata C., Riess A. G., Rozo E., 2013, Phys. Rep., 530, 87

White M., 2014, Mon. Not. R. Astron. Soc. 439, 3630

White M., 2015, Mon. Not. R. Astron. Soc. 450, 3822

White M., Padmanabhan N., 2017, Mon. Not. R. Astron. Soc. 471, 1167

White M., Carlstrom J. E., Dragovan M., Holzapfel W. L., 1999, Astrophys. J. , 514, 12

Yamamoto K., Nakamichi M., Kamino A., Bassett B. A., Nishioka H., 2006, Publ. Astron. Soc. Japan, 58, 93

Yoo J., Seljak U., 2015, Mon. Not. R. Astron. Soc. 447, 1789

Zaroubi S., Hoffman Y., 1996, Astrophys. J. 462, 25

Zel'dovich Y. B., 1970, Astron. Astrophys. , 5, 84 